\title{
Measurement of the air bubble size and velocity from micro air bubble generation (MBG) in diesel using optical methods
}

\author{
Bader A. Alfarraj ${ }^{1}$ (I) $\cdot$ Abdullah M. Alkhedhair ${ }^{1} \cdot$ Ahmed A. Al-Harbi $^{1} \cdot$ Wojciech Nowak $^{2} \cdot$ Saleh A. Alfaleh $^{1}$
}

Received: 27 September 2020 / Accepted: 19 October 2020 / Published online: 30 October 2020

(c) The Author(s) 2020

\begin{abstract}
In this paper, we determine the bubble size and velocity from air bubble generation (MBG) in a diesel using optical methods. A KTM Series Pump was used to generate micro air bubbles in diesel. The air bubble radius and velocity measurements can be useful parameters to optimize the bubble generation process. Two optical systems were used for measurement air bubble sizes and their velocities in diesel. First, the optical system without an objective lens was used to determine the velocity of air bubbles in diesel. Another optical system with a 10× objective lens was used to obtain the size distribution of air bubbles generated in diesel. An available optical system with a $10 \times$ objective lens can detect a bubble diameter greater than $3.3 \mu \mathrm{m}$ that air bubble images were processed using the ImageJ program. We measured the size distribution of air bubbles generated using the ImageJ program. The micro air bubble radius measured in diesel was found to be $6.26 \mu \mathrm{m}$ in the sample after a month from air bubble generation. In addition, the particle image velocimetry (PIV) technique was used to measure the velocity field. Then, we used the OpenPIV program for PIV image processing. The highest velocity distribution was determined to be $90 \mathrm{~mm} / \mathrm{s}$ for diesel without air bubbles and $20 \mathrm{~mm} / \mathrm{s}$ for diesel with air bubbles after a month of the bubble generation.
\end{abstract}

Keywords Bubble size $\cdot$ Bubble velocity $\cdot$ MBG $\cdot$ PIV

\section{Introduction}

The enhancement of engine performance and reduced pollutant emissions can impact positively on the environment and energy. Air bubble generation technology has been used to improve vehicle efficiency by reducing fuel consumption and reducing the fuel-air ratio [1]. Many studies were focused on the size distribution of air bubbles measurements to control air-fuel mixing by applying the liquid and airflow rates [2]. Bubble transportation and mechanisms of bubble breakup were also studied. Ghiji et al. investigated the effect of residual air bubbles on the diesel engine during the injection process [3]. In addition, the residual air bubble distribution

Bader A. Alfarraj

balfaraj55@gmail.com

1 National Center for Power Generation Tech. (NCPGT), Energy and Water Research Institute (EWRI), King Abdulaziz City for Science and Technology (KACST), P.O. Box 6086, Riyadh 11442, Saudi Arabia

2 Faculty of Energy and Fuels, AGH University of Science and Technology, A. Mickiewicza Av.30, 30-059 Krakow, Poland with diesel was experimentally and numerically studied on the nozzle spray [4]. However, pollutant emissions, such as NOx and SOx, can be decreased using bubble generation technology with fuel. There are three types of bubble generation: pressurized dissolution, gas-water circulation, and Venturi [5].

Air bubble size measurements are an important parameter to control the air bubble generator operation. The sizes of air bubbles have been measured in many studies [6-9]. Bubble coalescence and break-up effects make measuring bubble sizes challenging. Sherman Cheung et al. described bubble interaction mechanisms to understand some phenomena (e.g., bubble coalescence and break-up) by determining the bubble size and rising velocity [10]. They used two population balance models. Lau et al. [8] studied the behavior of bubbles during bubble coalescence and bubble break- up. The air bubble size was determined in a liquid medium using optical measurements [9]. Bubble coalescence or break-up can cause an overlapping bubble effect. The overlapping bubble effect is a major challenge when measuring the bubble size $[7,8]$. Image processing can be used to improve bubble detection for bubble size measurements. Some image processing algorithms have been developed to 
detect the actual bubble size $[7,8]$. Bubble detection and cluster splitting methods can be used to detect bubbles [6]. An example of a bubble detection method is the binary mask method to discriminate bubbles [11]. Examples of the cluster splitting method to detect bubbles are the threshold with the watershed algorithm [12] and the threshold with the inverted algorithm [6].

Measuring the velocity of bubbles is also an important factor to optimize the bubble generation process. The particle image velocimetry (PIV) technique was used to provide the velocity field of air bubbles [11,13-16]. It is an optical method and a nonintrusive measurement.

In addition, other techniques are used to analyze the size of microparticles. One of the methods is the nanoparticle tracking analysis (NTA) method to measure particle-by particle with high-resolution (i.e., (LM20) and (NS500) by NanoSight). Jeon et al. used NanoSight to measure bubble sizes [5]. No collection angle, wavelength, or refractive index of the solvent were required to measure the particle size in that solvent. Additionally, sample preparation was required. Another method, the dynamic light scattering (DLS) method was used to determine the particle size, molecular weight, concentration, and zeta potential (i.e., (ELSZ-2plus) by Otsuka Electronics, (NANOPHOX) by Sympatec, (SZ-100) by Horiba, and (LS 13 320) by Beckman Coulter). The bubble size and zeta potential were measured using the DLS method [17]. A wide range of particle sizes and concentrations can be measured using this method. A collection angle was required at $90^{\circ}$ and $173^{\circ}$. Microparticles can be also detected by weak scattering. The laser diffraction scattering (LDS) method was used to the measure particle size (i.e., (LS 13 320) by Beckman Coulter, (SALD $7100 \mathrm{HH})$ by Shimadzu, and (HELOS) by Sympatec). In addition, the resonant mass measurement (RMM) method was used to measure the number and size of particles (i.e., (Archimedes) by Malvern Instruments). The number of particles can be calculated in terms of milliliters. The bubble size was measured by Tesar using this method [18].

We studied the behavior of micro air bubbles in diesel. The number and size of the air bubbles in diesel were measured using the ImageJ program. The PIV technique was used to measure the velocity field using the OpenPIV program in MATLAB. The samples of air-bubbles in diesel were taken at different times during the bubble generation process.

\section{Experimental details}

A KTM Series Pump (model: KTM20N-000) was used to generate micro air bubbles in diesel. Four samples of airbubbles in diesel were taken at different times during the bubble generation process. The first sample was diesel without air bubbles before turning the bubble generator on. The second sample was taken after applying the bubble generator for five minutes. Micro air bubbles were homogeneously distributed through the whole diesel tank. The bubble generator system was turned off after $15 \mathrm{~min}$. Surely, micro air bubbles became relatively stable in diesel. Then, the third sample and the fourth sample were collected after the bubble generator was turned off by a half-hour and one month, respectively. All measurements were calculated in offline and were taken manually from the bubble generator.

\section{Experimental setup}

A schematic of the experimental setup used is shown in Fig. 1. A $532 \mathrm{~nm}-\mathrm{Nd}$ :YAG double-pulse laser (Spectra Physics, Quanta-Ray PIV-400, $10 \mathrm{~Hz}$ ) was used. The programmable timing unit (PTU 9, LaVision) was used to trigger synchronization of a CCD camera (FlowMaster II, by LaVision) and a Nd:YAG laser. The DaVis 7.2 software package (by LaVision) was used to control the PTU. Cylindrical lenses were used to expand the 2-D sheet beam. The laser sheet thickness was controlled using an iris diaphragm. The laser sheet was incident to a quartz cell cuvette $\left(10 \times 10 \times 45 \mathrm{~mm}^{3}\right.$, four clear windows). The scattering was focused using a Nikkon $50 \mathrm{~mm}$ (f\# 1.8) lens. A $532 \mathrm{~nm}$-bandpass filter was used to remove background and noise. The bandpass filter and lens were coupled to the CCD camera. An energy monitor (LM-PM10, By Coherent) was used to measure the laser energy. The laser energy was optimized to be $2.5 \mathrm{~mJ}$ for each pulse with a pulse repetition rate of $10 \mathrm{~Hz}$. A 10x microscope objective lens (with a numerical aperture (NA) of 0.25 , by AmScope) was used to detect micro air bubbles in diesel, as shown in Fig. 1b. The Nikkon lens was set to infinity focus when the microscope objective lens was coupled with the CCD camera.

The position and exposure time of the CCD camera were optimized using a calibration plate. The calibration plate was placed into a quartz cell cuvette, as shown in Fig. 2b. The laser sheet was aligned with the plane of the calibration plate. The exposure time was optimized using a calibration plate without a bandpass filter and beam laser. The exposure time was fixed to $4000 \mu$ s for satisfactory images in these experiments. A CCD camera recording mode was selected for single-frame mode.

Calibration of the camera is an important process for image processing. A camera measures the distance in pixels, not in millimeters. Most cameras have linear scaling, which show a linear relation between a measured distance in pixels and a true distance in millimeters at a $90^{\circ}$-viewing angle of the CCD camera with a calibration plate. A calibration plate is used for the scaling of the camera. The calibration plate contains black crosses and dots on a white background. The distance between the two end crosses in the calibration 
Fig. 1 Schematic of the used experimental setup used to measure the size and velocity of bubbles in diesel a without a microscope objective lens and b with a microscope objective lens

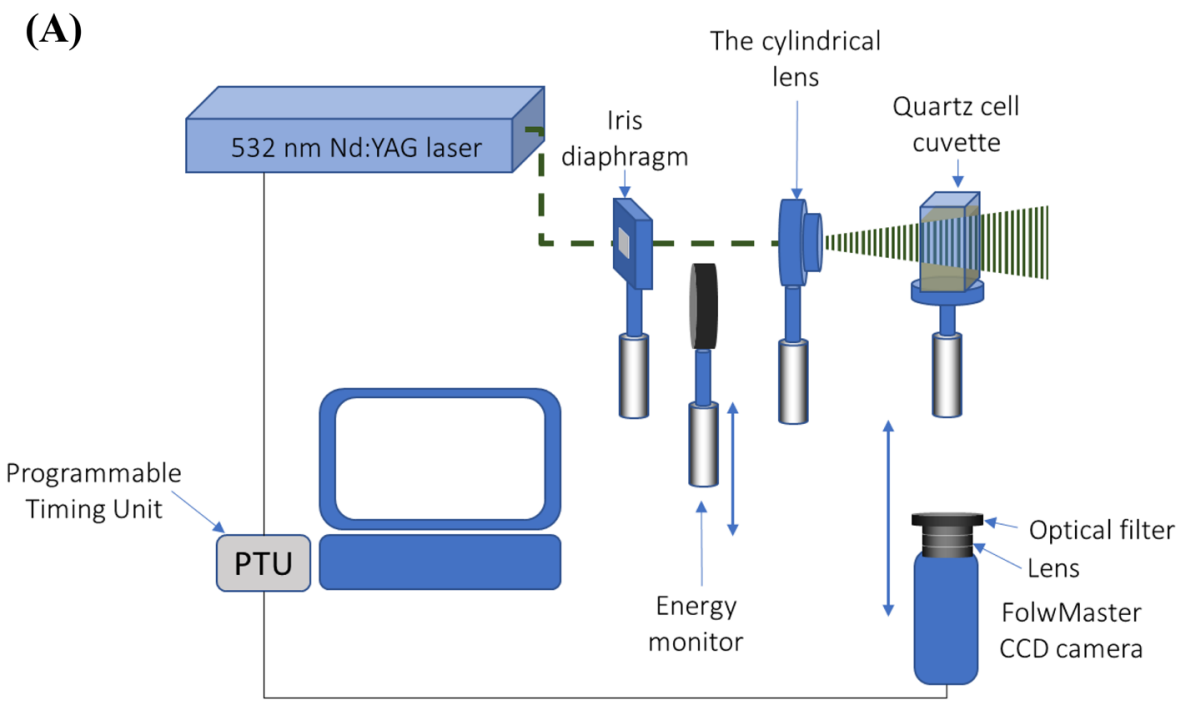

(B)

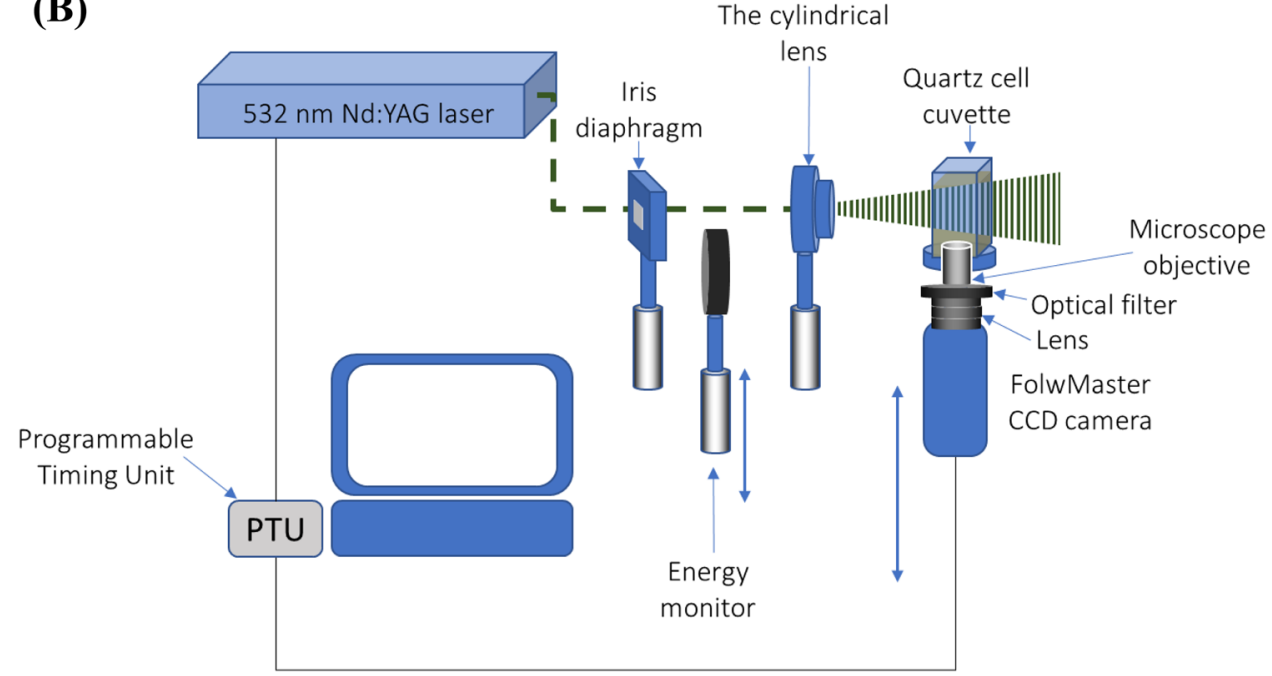

plate is $5 \mathrm{~mm}$. The length of one cross is $3 \mathrm{~mm}$, as shown in Fig. 2a. This was used in this study for micro air bubble velocity and size calculations.

The resolution for each optical system was calculated by measuring the length of the cross and the distance between two end crosses in pixels. The resolution is $3 \mathrm{~mm}$ divided by the cross length in pixels using the optical system with a $10 \times$ objective lens, as determined using MATLAB program. For the optical system without an objective lens, the resolution is $5 \mathrm{~mm}$ divided by the distance between two end crosses in pixels. The resolution was calculated to be $0.0827 \mathrm{~mm} /$ pixel $(\sim 82.70 \mu \mathrm{m} / \mathrm{pixel})$ for the optical system without an objective lens and $0.0033 \mathrm{~mm} /$ pixel $(\sim 3.30 \mu \mathrm{m} /$ pixel $)$ for the optical system with a $10 \times$ objective lens, as listed in Table 1. As a result, these optical systems cannot detect a micro air bubble diameter less than 82.70 and $3.30 \mu \mathrm{m}$ for the optical system without an objective lens and with $10 \times$ objective lens, respectively. These values will be used in the velocity and radius of the micro-air-bubble measurements. The positions of the camera were also marked for two optical systems after completing calibration. In Fig. 2b, the image window size was selected to be $6.12 \times 14.39$ for the optical system without an objective lens and $2.14 \times 1.57 \mathrm{~mm}^{2}$ for the optical system with a $10 \times$ objective lens.

\section{Measurements of the velocity of micro air bubbles in diesel}

The PIV technique was used to measure the velocity field of the micro air bubbles. The flow field was illuminated using a laser sheet. Two frames with a double-pulse laser were used with a known time between the two frames. There are many factors that impact the evaluation of velocity 
(A)

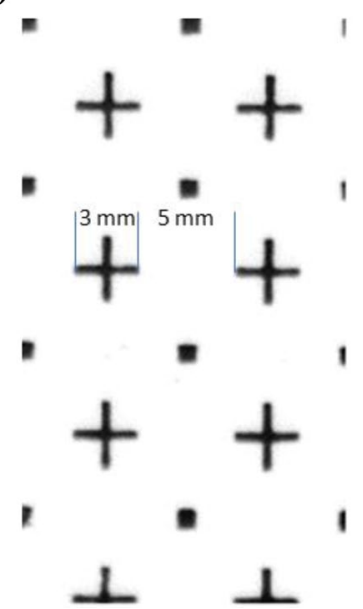

(B)

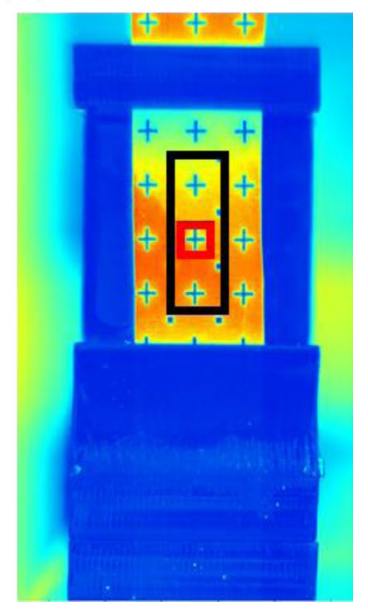

Fig. 2 Calibration plate: a marks on the calibration plate and $\mathbf{b}$ calibration plate placed in the sample holder

Table 1 Resolution and image window size of the optical systems

\begin{tabular}{llll}
\hline The kind of optical system & $\begin{array}{l}\text { Resolution } \\
(\mu \mathrm{m} / \text { pixel })\end{array}$ & $\begin{array}{l}\text { Image } \\
\text { window size } \\
\left(\mathrm{mm}^{2}\right)\end{array}$ \\
\hline 1 & Without an objective lens & 82.70 & $6.12 \times 14.39$ \\
2 & With a $10 \times$ objective lens & 3.30 & $2.14 \times 1.57$ \\
\hline
\end{tabular}

measurements. For example, the laser sheet thickness should cover the volume of the particle. A decrease in the numerical aperture (NA) and total magnification (M) of the optical system should increase the laser sheet thickness. The laser sheet thickness must be selected based on the particle size to reduce the noise levels. The PIV image will lose some information.

The single-frame mode was used to obtain PIV images with one exposure for every single frame using the DaVis 7.2 program. The PIV images were cropped to remove noise using MATLAB program. After cropping, image windows were analyzed by applying cross-correlation. The PIV crosscorrelation was performed using the OpenPIV 1.1 program for PIV image analysis [19]. The OpenPIV program is an open-source program, which was written in MATLAB program. The PIV cross-correlation subdivides images in the interrogation window size in the first frame. Each interrogation window in the first frame will be matched to identical particles in the second frame using cross-correlation. For example, the micro air bubble in the first frame was detected in the second frame with the highest correlation, as shown in Fig. 3c. The center of the interrogation window in the first frame was located at pixel $(307,209)$ at point $\left(x_{0}, y_{0}\right)$ at $t_{0}$. The identical particle was detected using cross-correlation

in the second frame at pixel $(308,189)$ at the point $\left(x_{1}, y_{1}\right)$ at $t_{1}$. The recording rate of the CCD camera was $5 \mathrm{~Hz}$ between two frames $\left(\mathrm{dt}=t_{1}-t_{0}=0.2 \mathrm{~s}\right)$. The displacement of the particle in the pixel unit was converted using the scale value in the OpenPIV program. The cross-correlation computes the displacement of the particle between two frames that have similar particles $\left(x_{1}-x_{0}, y_{1}-y_{0}\right)$.

The interrogation window should be selected based on the micro air bubble sizes and micro air bubble density in the interrogation window size, as shown by the white dashed lines in Fig. 3a. The interrogation window size was fixed to be $(16 \times 16)$ pixels for micro air bubbles in a diesel without an objective lens. In addition, the spacing or overlap size, which is the neighbor interrogation window, was fixed to be $(8 \times 8)$ pixels for micro air bubbles in a diesel without an objective lens. The interrogation window size should be selected to be greater than the spacing size. The velocity field of the micro air bubbles was calculated, as shown in Fig. 4. The velocity distribution of the micro air bubbles was shown in Fig. 5.

\section{Measurements of the size distribution of micro air bubbles in diesel}

An image processing and analysis software (ImageJ) program were used to measure the micro air bubble size distribution in diesel. The ImageJ program is public domain software and was written in Java by Wayne Rasband [20]. Each micro air bubble image contains separately processed and analyzed particles (e.g., the micro air bubble radius and the number of bubbles).

Four image processes were used to detect micro air bubbles in diesel, as shown in Fig. 6. The resolution of the optical system with a $10 \times$ objective lens was used as the calibration scale for the ImageJ program before image processing, as listed in Table 1. Micro air bubble detection and cluster splitting methods were used in this study. All images were converted to an 8-bit grayscale image, as shown in Fig. 6(2). The sharpening process was applied to improve the edge detection of particles, as shown in Fig. 6(3). The background was removed using a subtraction function via the process tab, as shown in Fig. 6(4). Threshold method was applied by converting the images to binary images, as shown in Fig. 6(5). The radius and number of the micro air bubbles were measured automatically using the "analyze particle" tool of the ImageJ program after image processing. 
Fig. 3 PIV cross-correlation technique: a the first image frame of the bubbles in diesel, b the next image frame of the bubbles in diesel $200 \mathrm{~ms}$ after the first frame, $\mathbf{c}$ cross-correlation of one of the interrogation windows

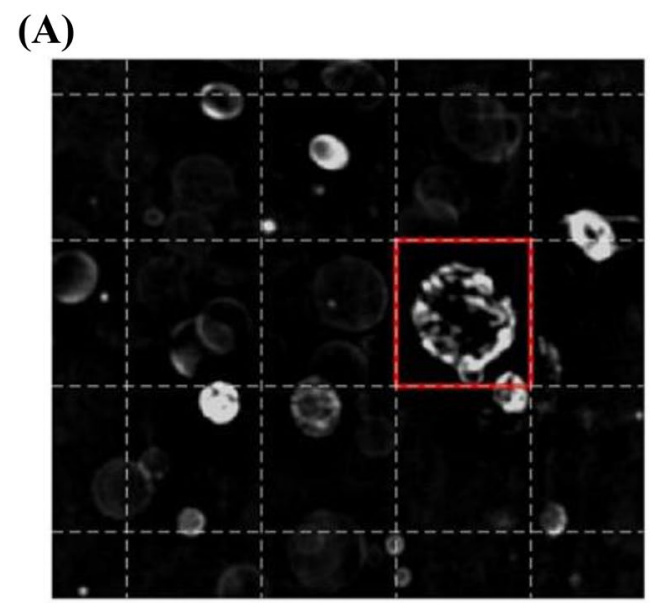

(B)

(C)
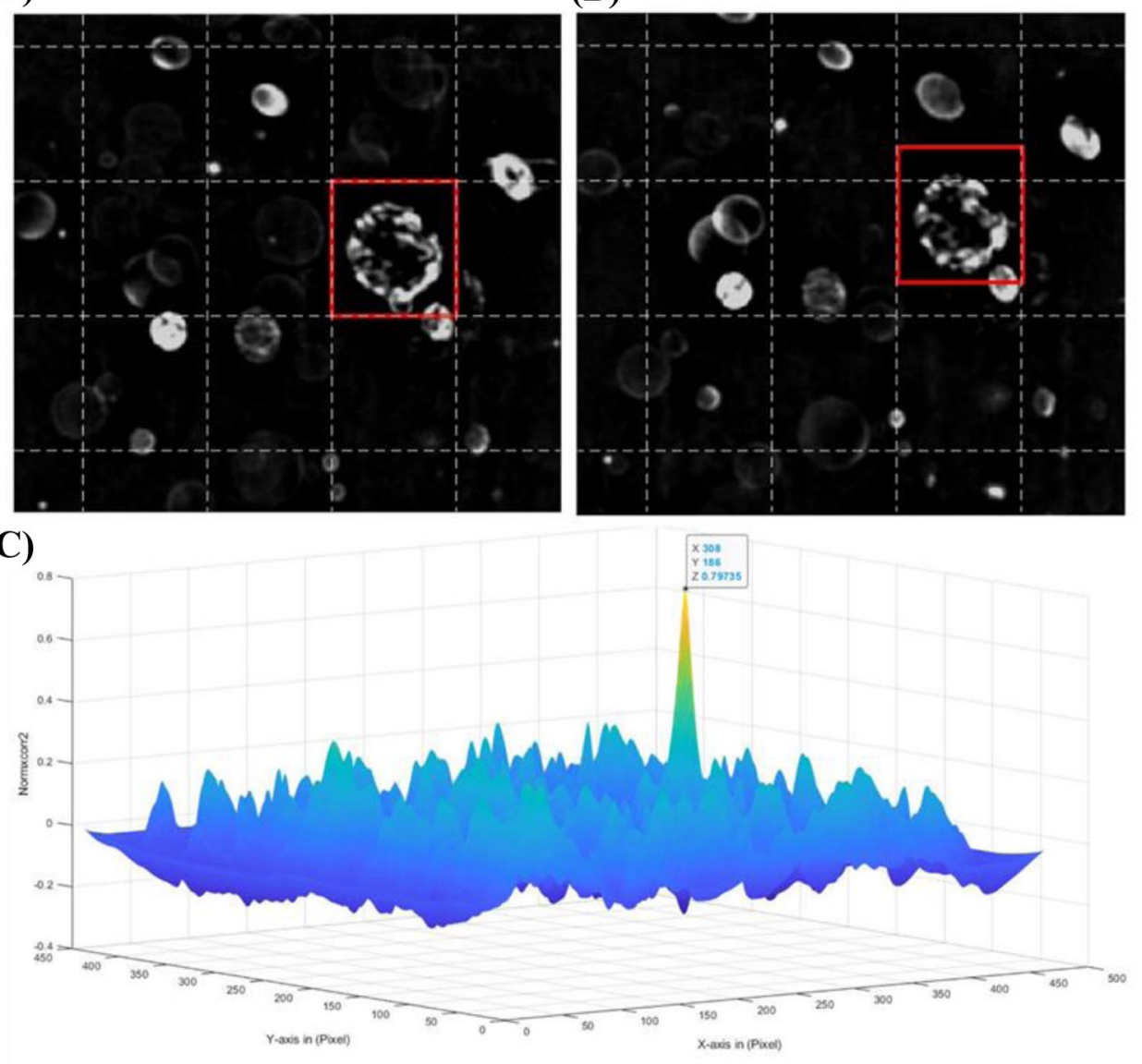

(A)

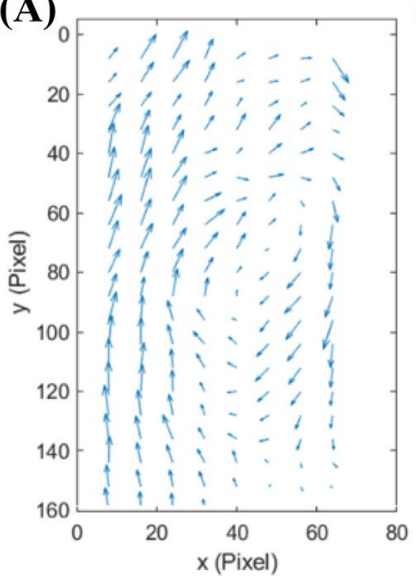

(B)

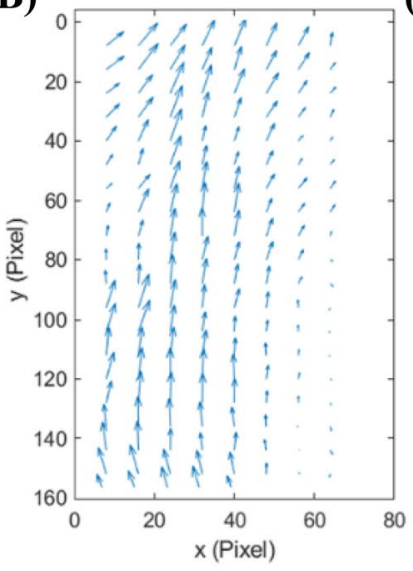

(C)

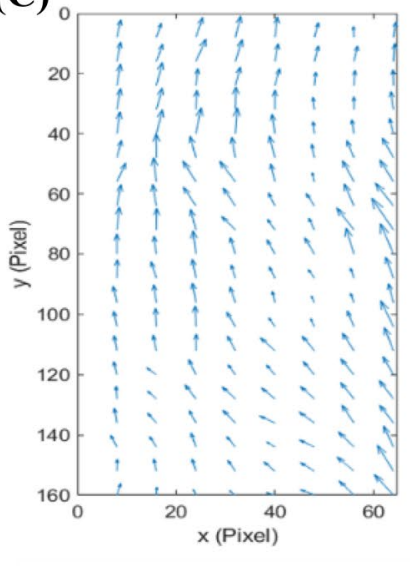

(D)

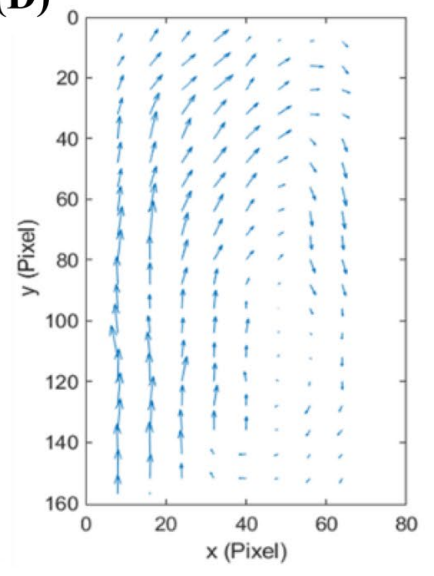

Fig. 4 The velocity map of the bubbles in diesel at different times through bubble generation: a diesel without bubbles, $\mathbf{b}$ during bubble generation, $\mathbf{c}$ bubbles in diesel after a half hour, and $\mathbf{d}$ bubbles in diesel after a month using the optical system without an objective lens

\section{Results and discussion}

The 300 micro air bubble images of each sample were used to measure the micro air bubble velocity and size for the four samples of micro air bubbles in diesel. The optical system without an objective lens was used to determine the velocity of micro air bubbles in diesel. The optical system with a $10 \times$ objective lens was used to determine the size distribution of micro air bubbles in diesel. 


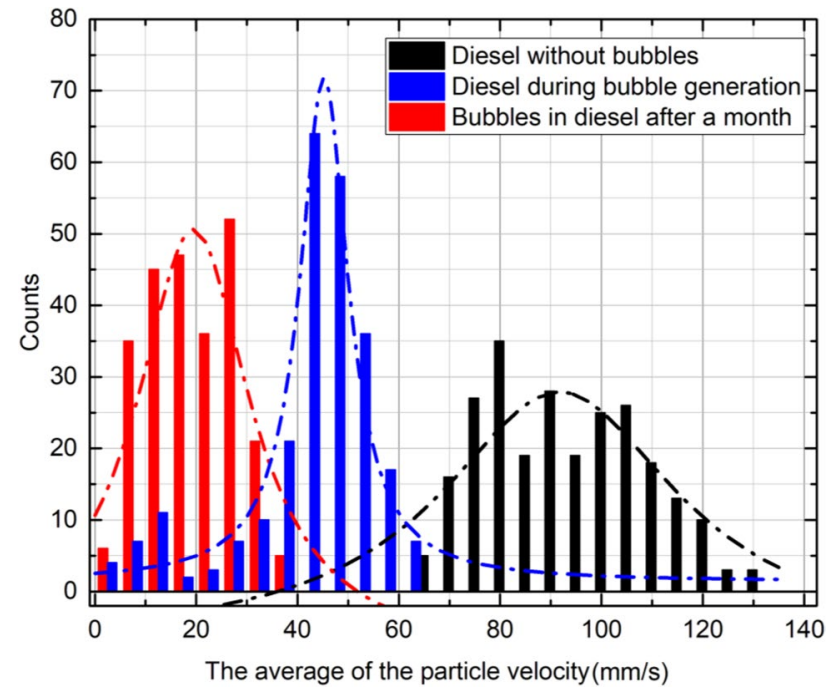

Fig. 5 Distribution of the velocity of bubbles in diesel at different times through bubble generation

The PIV cross-correlation was performed using 300 PIV images. An average of velocity vectors was the sum of the velocity vectors divided by the number of PIV images for each spacing size (in 300 PIV images). This function was useful to reduce the background noise level and improve the accuracy of velocity measurement [14]. The diesel particles and micro air bubbles in diesel velocity vectors were calculated for the optical system without an objective lens at different times through bubble generation, as shown in Fig. 4. The fluid motion rotated in the vortex ring at diesel without micro air bubbles, as shown in Fig. 4a. In diesel, during bubble generator operation for 5 minutes, the fluid motion moved in one direction with the rising bubble direction. This is the first step for bubble nucleation through bubble generation in the early period of bubble generation, as shown in Fig. 4b. The direction of micro air bubbles movement mostly contained rising micro air bubbles, with a few random collisions micro air bubble coalescence or break-up for the third sample, as shown in Fig. 4c. In micro air bubbles in diesel a month after the bubble generator was turned off, the fluid motion was in a steady state without collisions, as shown in Fig. 4d. The velocities of the micro air bubbles in diesel for a month, after the bubble generator was turned off, moved slowly compared with the velocities of the diesel particles and diesel during the bubble generation process. This is a good arrangement in which the velocity depends on the particle diameter according to Stokes' law.

Each sample had $(8 \times 20)$ velocity vectors. The velocity distribution of the particles was obtained for different times through bubble generation, as shown in Fig. 5. An average of 160 velocity vectors for each sample was used to plot the velocity distribution of the particles. The highest velocity distribution was found to be 90,45 , and $20 \mathrm{~mm} / \mathrm{s}$ for diesel without bubbles, diesel during the bubble generation process, and micro air bubbles in diesel one month after the bubble generator was turned off, respectively.

The radius of micro air bubbles was measured using the ImageJ program for diesel during the bubble generation process; micro air bubbles in diesel were measured a halfhour after the bubble generator was turned off, and micro air bubbles in diesel were measured a month after the bubble generator was turned off. The radius and number of micro air bubbles were calculated in a $2.14 \times 1.57 \mathrm{~mm}^{2}$ image window size. The micro air bubble radius can be detected using the optical system with a $10 \times$ objective lens. The micro air bubble in diesel was approximately $6.26 \mu \mathrm{m}$ from micro air bubbles in diesel one month after the bubble generator was turned off, as shown in Fig. 7. The highest micro air bubble radius distribution was found to be approximately $10 \mu \mathrm{m}$. An average of the number and size micro-air-bubbles of the 300 images was used to plot the micro-air-bubble radius and number distribution.
(1)

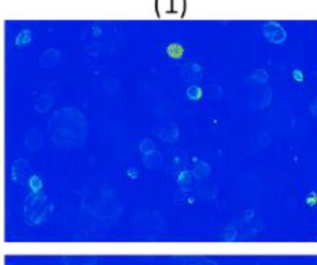

(B)

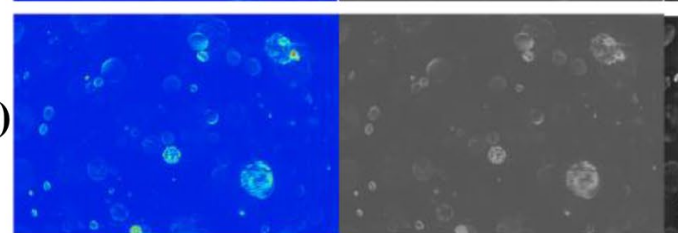

(2)

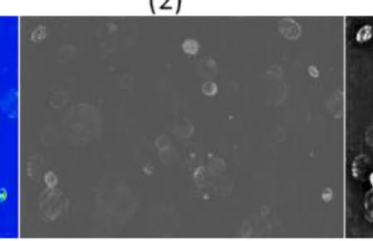

(3)

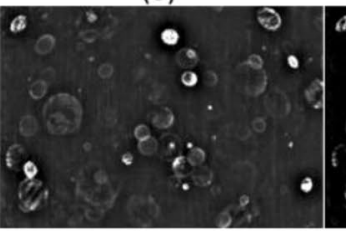

(4)

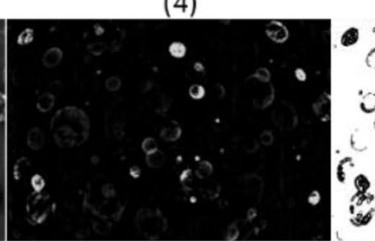

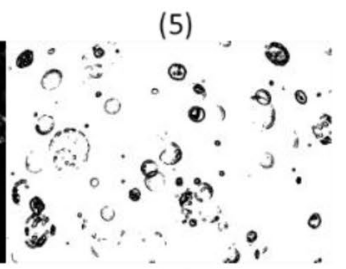

(5)
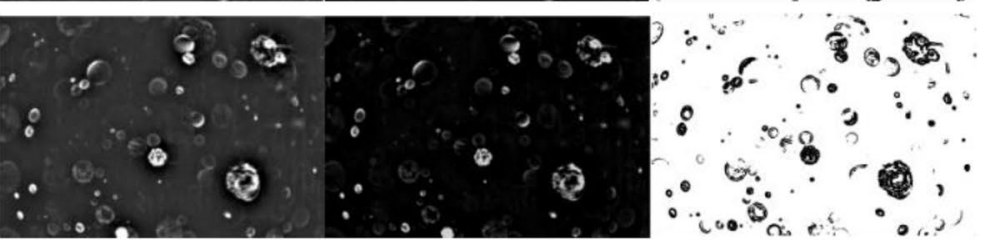

Fig. 6 Image processing of bubbles for radius measurement: $\mathbf{a}$ at during bubble generation and $\mathbf{b}$ bubbles in diesel after a half-hour using the optical system with $10 \times$ objective lens 

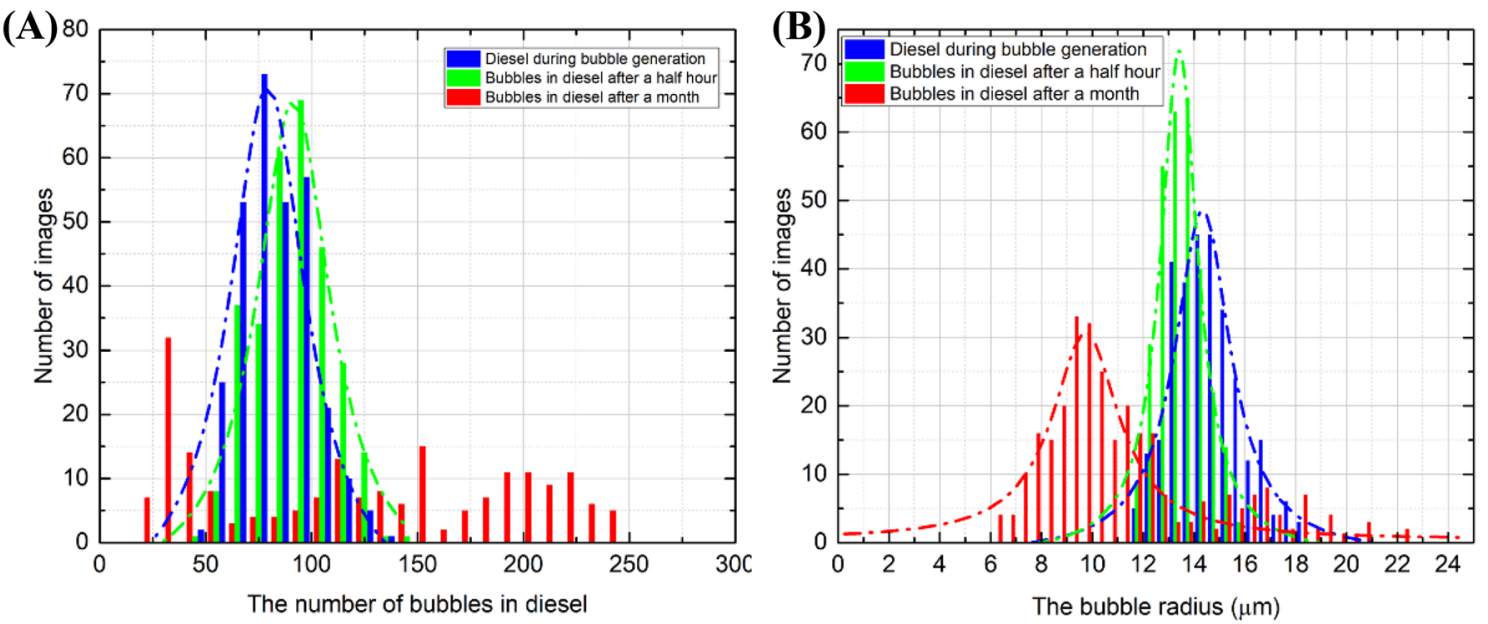

Fig. 7 Distribution of the average number (a) and radius (b) of the bubbles in diesel at different times through bubble generation using the optical system with a $10 \times$ objective lens (300 images)

The highest micro air bubble radius distribution in diesel a month after the bubble generator was turned off was smaller than the highest distribution of the micro air bubble radius in diesel a half-hour after the bubble generator was turned off and during the bubble generation process. Generally, the number of micro air bubbles increases with decreasing micro air bubble radius during the bubble generation process and for micro air bubbles in diesel a halfhour after the bubble generator was turned off. An increase in the micro air bubble radius will increase the velocity of the micro air bubbles according to the results presented in Figs. 5 and 7.

\section{Conclusions}

In this study, the size and velocity of the micro air bubbles in diesel were successfully measured. The samples of air bubbles in diesel were taken at different times during and after the bubble generation process using a KTM Series Pump. The size of air bubbles in diesel was measured using the ImageJ program for image processing. The PIV technique was used to measure the velocity field using the OpenPIV program in MATLAB. The highest air bubble velocity was found with a large air bubble size. The velocity of the diesel particles without air bubbles was $90 \mathrm{~mm} / \mathrm{s}$. A month after turning off the bubble generator, the air bubbles were still suspended in diesel due to their micro-level size, and the lowest velocity was found to be $20 \mathrm{~mm} / \mathrm{s}$. The highest velocity distribution was found at $45 \mathrm{~mm} / \mathrm{s}$ for diesel during the bubble generation process.
In addition, the highest radius of air bubble measured was $10 \mu \mathrm{m}$. The micro air bubble radius was approximately $6.26 \mu \mathrm{m}$ for micro air bubbles in diesel one month after the bubble generator was turned off.

Acknowledgments The authors would like to express their gratitude to the National Center for Power Generation Tech. (NCPGT), Energy and Water Research Institute (EWRI), King Abdulaziz City for Science and Technology (KACST) for supporting this project.

Open Access This article is licensed under a Creative Commons Attribution 4.0 International License, which permits use, sharing, adaptation, distribution and reproduction in any medium or format, as long as you give appropriate credit to the original author(s) and the source, provide a link to the Creative Commons licence, and indicate if changes were made. The images or other third party material in this article are included in the article's Creative Commons licence, unless indicated otherwise in a credit line to the material. If material is not included in the article's Creative Commons licence and your intended use is not permitted by statutory regulation or exceeds the permitted use, you will need to obtain permission directly from the copyright holder. To view a copy of this licence, visit http://creativecommons.org/licenses/by/4.0/.

\section{References}

1. Nakatake, Y., Kisu, S., Shigyo, K., Eguchi, T., Watanabe, T.: Effect of nano air-bubbles mixed into gas oil on common-rail diesel engine. Energy. 59, 233-239 (2013)

2. Huang, J., Sun, L., Liu, H., Mo, Z., Tang, J., Xie, G., Du, M.: A review on bubble generation and transportation in Venturi-type bubble generators. Exp. Comput. Multiph. Flow. 2, 123-134 (2020). https://doi.org/10.1007/s42757-019-0049-3

3. Ghiji, M., Goldsworthy, L., Garaniya, V., Brandner, P.A., Hield, P., Novozhilov, V., Moinuddin, K., Joseph, P.: Effect of residual air bubbles on diesel spray structure at the start of injection. Fuel 241, 25-32 (2019). https://doi.org/10.1016/j.fuel.2018.12.013 
4. Guo, G., He, Z., Lai, M.C., Duan, X., Leng, X., Duan, L., Chen, Z.: Optical experiment and Large Eddy Simulation on effects of in-nozzle stagnant air bubbles and diesel on near-nozzle spray structure variation in diesel injector. Fuel 255, 115721 (2019). https://doi.org/10.1016/j.fuel.2019.115721

5. Jeon, S.-Y., Yoon, J.-Y., Jang, C.-M.: Bubble size and bubble concentration of a microbubble pump with respect to operating conditions. Energies. 11, 1864 (2018)

6. Gordiychuk, A., Svanera, M., Benini, S., Poesio, P.: Size distribution and Sauter mean diameter of micro bubbles for a Venturi type bubble generator. Exp. Therm. Fluid Sci. 70, 51-60 (2016)

7. Lau, Y.M., Sujatha, K.T., Gaeini, M., Deen, N.G., Kuipers, J.A.M.: Experimental study of the bubble size distribution in a pseudo-2D bubble column. Chem. Eng. Sci. 98, 203-211 (2013)

8. Lau, Y.M., Deen, N.G., Kuipers, J.A.M.: Development of an image measurement technique for size distribution in dense bubbly flows. Chem. Eng. Sci. 94, 20-29 (2013)

9. Gaillard, T., Honorez, C., Jumeau, M., Elias, F., Drenckhan, W.: A simple technique for the automation of bubble size measurements. Colloids Surfaces A Physicochem. Eng. Asp. 473, 68-74 (2015). https://doi.org/10.1016/j.colsurfa.2015.01.089

10. Cheung, S.C.P., Yeoh, G.H., Tu, J.: A review of population balance modelling for isothermal bubbly flows. J. Comput. Multiph. Flows. 1, 161-199 (2009)

11. Cerqueira, R.F.L., Paladino, E.E., Ynumaru, B.K., Maliska, C.R.: Image processing techniques for the measurement of two-phase bubbly pipe flows using particle image and tracking velocimetry (PIV/PTV). Chem. Eng. Sci. 189, 1-23 (2018)

12. Gaillard, T., Roché, M., Honorez, C., Jumeau, M., Balan, A., Jedrzejczyk, C., Drenckhan, W.: Controlled foam generation using cyclic diphasic flows through a constriction. Int. J. Multiph. Flow. 96, 173-187 (2017)

13. Todd, D.R., Hassan, Y.A.: Analysis of bubbly flow using particle image velocimetry. In: International Conference on Nuclear Engineering (ICONE 9.). pp. 8-12 (2001). https://www.osti.gov/ etdeweb/servlets/purl/20241582. Accessed 14 July 2020

14. Ziegenhein, T., Garcon, M., Lucas, D.: Particle tracking using micro bubbles in bubbly flows. Chem. Eng. Sci. 153, 155-164 (2016)

15. Ishikawa, M., Irabu, K., Teruya, I., Nitta, M.: PIV measurement of a contraction flow using micro-bubble tracer. In: Journal of Physics: Conference Series. pp. 82-86 (2009). https://iopscience .iop.org/article/10.1088/1742-6596/147/1/012010/meta. Accessed 1 July 2020

16. Lindken, R., Merzkirch, W.: A novel PIV technique for measurements in multiphase flows and its application to two-phase bubbly flows. Exp. Fluids. 33, 814-825 (2002)

17. Ushikubo, F.Y., Furukawa, T., Nakagawa, R., Enari, M., Makino, Y., Kawagoe, Y., Shiina, T., Oshita, S.: Evidence of the existence and the stability of nano-bubbles in water. Colloids Surfaces A Physicochem. Eng. Asp. 361, 31-37 (2010)

18. Tesavr, V.: Microbubble generation by fluidics, Part II: Bubble formation mechanism. In: Proc. of Colloquium Fluid Dynamics. pp. 1-20 (2012). http://crom.it.cas.cz/files/u1868/tesar1_F.pdf. Accessed 9 Jan 2020

19. OpenPIV Group.: OpenPIV program. www.openpiv.net. Accessed 14 July 2020

20. Wayne Rasband: ImageJ program. https://rsbweb.nih.gov/ ij/. Accessed 14 July 2020 